\title{
A simple and eco-friendly synthesis of 3-indolyl-3-hydroxy oxindoles and 11-indolyl-11 $H$-indeno[1,2-b]quinoxalin-11-ols in aqueous media
}

\author{
G. Shanthi, N. Vidhya Lakshmi, and Paramasivan T. Perumal* \\ Organic Chemistry Division, Central Leather Research Institute, Adyar, \\ Chennai 600 020, India \\ E-mail:ptperumal@gmail.com
}

\begin{abstract}
A simple and convenient method for the synthesis of 3-indolyl-3-hydroxy oxindoles and 11indolyl-11 $\mathrm{H}$-indeno[1,2-b] quinoxalin-11-ols catalyzed by $\mathrm{K}_{2} \mathrm{CO}_{3}$ in aqueous media is described.
\end{abstract}

Keywords: Isatin, $\mathrm{K}_{2} \mathrm{CO}_{3}$, oxindole derivatives, indenoquinoxalines, water mediated reaction

\section{Introduction}

3-Substituted 3-hydroxyindolin-2-ones are important substrates for studies of biological activities as well as useful synthetic intermediates for drug candidates and alkaloids. The development of practical methods for their preparation is of interest. 3-Substituted 3hydroxyoxindoles are encountered in a large variety of natural products with a wide spectrum of biological activities, such as convolutamydines, ${ }^{1}$ donaxaridines, ${ }^{2}$ maremycins, ${ }^{3}$ dioxibrassinines, ${ }^{4}$ celogentin $\mathrm{K}^{5}{ }^{3} 3$ '-hydroxy glucoisatisin, ${ }^{6}$ and TMC-95A. ${ }^{7}$ 3-Alkenyl- and 3-aryl-substituted 3hydroxyindoles, ${ }^{8}$ and their derivatives ${ }^{9}$ have been used in a number of recent pharmaceutical studies. The formation of quaternary carbon centers via addition of nucleophiles to ketone derivatives still constitutes a major challenge for synthetic chemistry. Recently, organic reactions in water have attracted great interest in organic synthesis because of its cost, safety and environmental concern. ${ }^{10}$ Therefore, the development of an efficient synthetic methodology to form a carbon-carbon bond in water appears to be very important.

The synthesis of monosubstituted 3-indolyl-3-hydroxy oxindoles by Friedel-Crafts reaction of indoles with electron-deficient carbonyl compounds such as isatins will be one of the synthetically useful transformations. ${ }^{11}$ Musabekova et al. have reported the condensation reaction of isatin with 2-methylindole in presence of acetic acid to afford 3-hydroxyindolines. ${ }^{12 a}$ Rama Rao et al. ${ }^{12 \mathrm{~b}}$ reported the synthesis of 3-indolyl-3-hydroxy oxindoles using $\beta$-cyclodextrin in good yields. The scope of the reaction was limited to isatin only and the reaction was carried 
out at $50{ }^{\circ} \mathrm{C}$. Thus, there is a need to develop a generally applicable, mild and environmentally benign practical methodology at ambient temperature.

\section{Results and Discussion}

As part of our current studies on the design of new routes for the preparation of biologically active heterocyclic compounds, ${ }^{13}$ we herein disclose a simple and convenient method for the efficient synthesis of 3-indolyl-3-hydroxy oxindoles in water catalyzed by $\mathrm{K}_{2} \mathrm{CO}_{3}$ (Scheme 1).

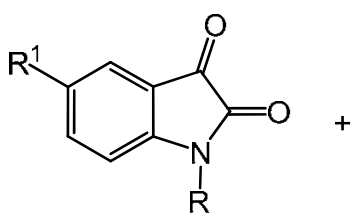

1<smiles>[R]c1ccc2cc([R])[nH]c2c1</smiles>

2

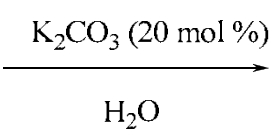

$\mathrm{H}_{2} \mathrm{O}$

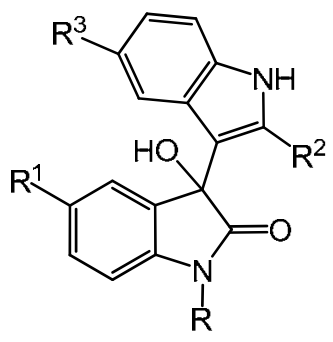

3

\section{Scheme 1}

In order to study the scope and limitations of the reaction, various bases, including sodium acetate, potassium acetate, ammonium acetate, basic alumina, potassium carbonate and sodium carbonate and different solvent systems were investigated. The best overall yield (91\%) was obtained with potassium carbonate in water. Optimal result was obtained using $20 \mathrm{~mol} \%$ of $\mathrm{K}_{2} \mathrm{CO}_{3}$.

The experiment was conducted with isatin $\mathbf{1}$ and indole $\mathbf{2}$ in the presence of catalytic amount of $\mathrm{K}_{2} \mathrm{CO}_{3}(20 \mathrm{~mol} \%)$ in water. The reaction proceeded spontaneously at ambient temperature and was completed within $1 \mathrm{~h}$. The isolation of product was straightforward as the solid precipitated on completion of the reaction. The precipitated solid was filtered, dried and washed with $20 \%$ ethyl acetate in petroleum ether to afford 3-indolyl-3-hydroxy oxindole 3 . The pure product 3 was isolated up to $91 \%$ yield without column chromatography. The result provided the incentive for further study of reactions with various other isatin derivatives and substituted indoles to furnish the corresponding 3-indolyl-3-hydroxy oxindoles.

The structures of compounds 3a-n were confirmed by IR, ${ }^{1} \mathrm{H}$ and ${ }^{13} \mathrm{C}$ NMR spectroscopy, mass spectrometry and elemental analysis. The mass spectrum of 3c displayed the molecular ion $\left[\mathrm{M}^{+}\right]$peak at $m / z$ 295. The ${ }^{1} \mathrm{H}$ NMR spectrum of 3c exhibited broad singlets due to -OH and -NH protons at $\delta 6.32,10.30$ and $10.81\left(\mathrm{D}_{2} \mathrm{O}\right.$ exchangeable) respectively. Signals at $\delta 74.9$ (quaternary carbon) and $178.4(-\mathrm{C}=\mathrm{O})$ in the ${ }^{13} \mathrm{C}$ spectrum confirmed the formation of the product. 
Table 1. Synthesis of 3-indolyl-3-hydroxy oxindoles

\begin{tabular}{ccccccc}
\hline $\mathrm{R}$ & $\mathrm{R}^{1}$ & $\mathrm{R}^{2}$ & $\mathrm{R}^{3}$ & Product (3) & Time(min.) & Yield(\%) \\
\hline $\mathrm{H}$ & $\mathrm{H}$ & $\mathrm{H}$ & $\mathrm{H}$ & $\mathbf{3 a}$ & 60 & 91 \\
$\mathrm{H}$ & $\mathrm{H}$ & methyl & $\mathrm{H}$ & $\mathbf{3 b}$ & 70 & 92 \\
$\mathrm{H}$ & $\mathrm{H}$ & $\mathrm{H}$ & methoxy & $\mathbf{3 c}$ & 60 & 93 \\
$\mathrm{H}$ & $\mathrm{H}$ & $\mathrm{H}$ & bromo & $\mathbf{3 d}$ & 120 & 85 \\
Allyl & $\mathrm{H}$ & $\mathrm{H}$ & $\mathrm{H}$ & $\mathbf{3 e}$ & 90 & 90 \\
Allyl & $\mathrm{H}$ & methyl & $\mathrm{H}$ & $\mathbf{3 f}$ & 80 & 92 \\
Allyl & $\mathrm{H}$ & $\mathrm{H}$ & bromo & $\mathbf{3 g}$ & 140 & 83 \\
Benzyl & $\mathrm{H}$ & $\mathrm{H}$ & $\mathrm{H}$ & $\mathbf{3 h}$ & 60 & 90 \\
Benzyl & $\mathrm{H}$ & methyl & $\mathrm{H}$ & $\mathbf{3 i}$ & 70 & 92 \\
Benzyl & $\mathrm{H}$ & $\mathrm{H}$ & methoxy & $\mathbf{3 j}$ & 60 & 91 \\
Benzyl & $\mathrm{H}$ & $\mathrm{H}$ & bromo & $\mathbf{3 k}$ & 120 & 85 \\
$\mathrm{H}$ & chloro & $\mathrm{H}$ & $\mathrm{H}$ & $\mathbf{3 l}$ & 90 & 89 \\
$\mathrm{H}$ & chloro & methyl & $\mathrm{H}$ & $\mathbf{3 m}$ & 80 & 94 \\
$\mathrm{H}$ & chloro & $\mathrm{H}$ & methoxy & $\mathbf{3 n}$ & 70 & 94 \\
\hline
\end{tabular}

${ }^{\mathrm{a}}$ Isolated yield confirmed through IR, NMR, mass spectrometry.

Based on the above results, we have proposed a plausible mechanism in Scheme 2. Initially isatin reacts with indole in presence of $\mathrm{K}_{2} \mathrm{CO}_{3}$ in water to give the transition state 2a which leads to the formation of the product 3-indolyl-3-hydroxy oxindole 3.<smiles></smiles>

\section{Scheme 2}

Substituted quinoxaline derivatives are pharmacologically important compounds. ${ }^{14 a}$ Although rarely described in nature, various antibiotics such as echinomycin, levomycin and actinoleutin possessing a quinoxaline ring are known to inhibit the growth of gram-positive bacteria and are also active against various transplantable tumors. ${ }^{14}$ Azizian et al. ${ }^{15}$ have utilized the carbonyl group of the indenoquinoxaline in a reaction with 4-hydroxyproline for the 
synthesis of pyrrolyl indenoquinoxaline derivatives. Azizian et al. ${ }^{16}$ have synthesised new spiro indenoquinoxaline-pyrazolines from indenoquinoxaline and acetophenone. Recently, we reported the synthesis of spiroindenoquinoxaline by the three-component condensation of indenoquinoxaline, malononitrile and 1-phenyl-3-methyl pyrazolon-5-one. ${ }^{13 a}$ However, there have been no examples for the synthesis of 11-indolyl-11H-indeno[1,2-b]quinoxalin-11-ols reported so far. Under similar conditions, we investigated the reaction of indenoquinoxaline 4 with indoles to synthesize the corresponding 11-indolyl-11H-indeno[1,2-b]quinoxalin-11-ols 5 . The reaction was rather slow and the best overall yield (85\%) was obtained when the reaction was carried out in water : ethanol (1:1) (Scheme 3).

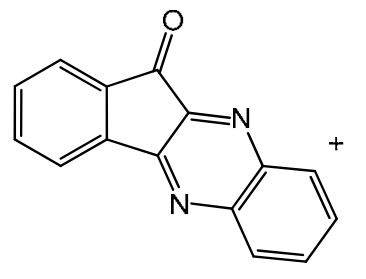

4<smiles>[R]c1ccc2[nH]c([R])cc2c1</smiles>

2

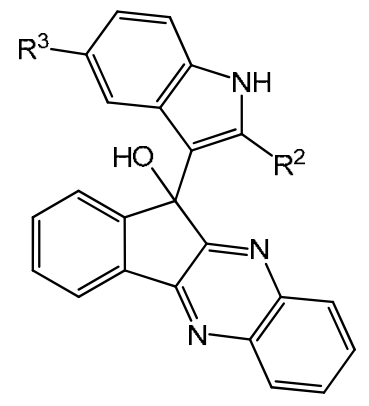

5

\section{Scheme 3}

The structures of compounds 5a-c were confirmed by spectroscopic and elemental data. The mass spectrum of $\mathbf{5 a}$ displayed the molecular ion $\left[\mathrm{M}^{+}\right]$peak at $\mathrm{m} / \mathrm{z} 350$. The ${ }^{1} \mathrm{H}$ NMR of $5 \mathbf{a}$ displayed broad singlets at $\delta 6.61$ and 11.03 due to $-\mathrm{OH}$ and $-\mathrm{NH}$ protons $\left(\mathrm{D}_{2} \mathrm{O}\right.$ exchangeable) respectively. A distinguishing signal at $\delta 77.1$ (quaternary carbon) confirmed the formation of the product.

Table2. Synthesis of 11-indolyl-11H-indeno[1,2-b]quinoxalin-11-ols

\begin{tabular}{ccccc}
\hline $\mathrm{R}^{2}$ & $\mathrm{R}^{3}$ & Product (5) & Time (min.) & Yield (\%) $^{\mathrm{a}}$ \\
\hline $\mathrm{H}$ & $\mathrm{H}$ & $\mathbf{5 a}$ & 240 & 85 \\
$\mathrm{H}$ & methoxy & $\mathbf{5 b}$ & 240 & 83 \\
$\mathrm{H}$ & bromo & $\mathbf{5 c}$ & 280 & 78 \\
\hline
\end{tabular}

${ }^{a}$ Isolated yield confirmed through IR, NMR, Mass spectrometry

\section{Conclusions}

In summary, we have developed a green approach to the synthesis of various 3-indolyl-3hydroxy oxindoles and 11-indolyl-11H-indeno[1,2-b]quinoxalin-11-ols. The present protocol 
offers several advantages such as (a) clean and simple reaction procedure (b) isolation of products without employing purification methods like column chromatography (c) use of ecofriendly catalyst. Biological evaluation of these derivatives is underway.

\section{Experimental Section}

General Procedures. IR measurements were done as $\mathrm{KBr}$ pellets for solids using Perkin Elmer Spectrum RXI FT-IR. The ${ }^{1} \mathrm{H}$ and ${ }^{13} \mathrm{C}$ NMR spectra were recorded in DMSO- $\mathrm{d}_{6}$ with JEOL $500 \mathrm{MHz}$, Bruker $500 \mathrm{MHz}$ and Bruker $300 \mathrm{MHz}$ high resolution NMR spectrometer. DMSO- $\mathrm{d}_{6}$ was used as the solvent for the NMR spectral measurements and spectra were recorded in ppm with TMS as internal standard. Multiplicities are abbreviated as follows: singlet (s), doublet (d), triplet $(\mathrm{t})$, multiplet $(\mathrm{m})$, and broad (br). The mass spectra were analyzed by using a Electrospray Ionisation Method with Thermo Finnigan Mass spectrometer. Melting points were determined in capillary tubes and are uncorrected. Elemental analyses were recorded using a Thermo Finnigan FLASH EA $1112 \mathrm{CHN}$ analyzer. Analytical TLC was performed on precoated plastic sheets of silica gel G/UV-254 of $0.2 \mathrm{~mm}$ thickness (Macherey-Nagel).

\section{General procedure for the synthesis of 3-Indolyl-3-hydroxy oxindoles}

To the reaction mixture containing isatin $\mathbf{1}(1 \mathrm{mmol})$ and indole $2(1 \mathrm{mmol})$ in water $(5 \mathrm{~mL})$, catalytic amount of potassium carbonate $(20 \mathrm{~mol} \%)$ was added and stirred at room temperature for about $1 \mathrm{~h}$. The precipitated solid was filtered, dried and washed with $20 \%$ ethyl acetate in petroleum ether to afford the pure product in $91 \%$ yield.

\section{General procedure for the synthesis of 11-indolyl-11H-indeno[1,2-b]quinoxalin-11-ols}

To the reaction mixture containing indenoquinoxaline $4(1 \mathrm{mmol})$ and indole $2(1 \mathrm{mmol})$ in ethanol:water mixture (1:1), catalytic amount of potassium carbonate (20 mol \%) was added and stirred at room temperature for about $4 \mathrm{~h}$. The precipitated solid was filtered, dried and washed with $20 \%$ ethyl acetate in petroleum ether to afford the pure product in $85 \%$ yield.

3-Hydroxy-3-(1H-indol-3-yl)indolin-2-one (3a). White solid; $\mathrm{mp}: 294-296{ }^{\circ} \mathrm{C} ; \mathrm{R}_{\mathrm{f}} 0.25$ (50\% AcOEt/Petroleum ether); IR (neat): 3401, 3054, 2926, $1706 \mathrm{~cm}^{-1} ;{ }^{1} \mathrm{H} \mathrm{nmr}\left(500 \mathrm{MHz}, \mathrm{DMSO}-\mathrm{d}_{6}\right)$ : $\delta 6.34$ (1 H, s, OH, $\mathrm{D}_{2} \mathrm{O}$ exchangeable), 6.84-6.9 (3 H, m), 6.99 (1 H, t, J 7.65), 7.04 (1 H, d, J 2.3), 7.21 (2 H, t, J 8.4), 7.31 (2 H, t, J 9.1), 10.32 (1 H, s, NH), $10.96(1 \mathrm{H}, \mathrm{s}, \mathrm{NH}) ;{ }^{13} \mathrm{C} \mathrm{nmr}$ (125 MHz, DMSO-d $)_{6}$ : 75.4 (s), 110.1 (d), 112.0 (d), 115.9 (s), 118.9 (d), 120.8 (d), 121.5 (d), 122.2 (d), 124.0 (d), 125.2 (d), 125.4 (s), 129.5 (d), 133.9 (s), 137.3 (s), 142.1 (s), 178.9 (s); MS (EI): $\mathrm{m} / \mathrm{z}=265.08\left[\mathrm{M}^{+}\right]$. Anal. Calcd for $\mathrm{C}_{16} \mathrm{H}_{12} \mathrm{~N}_{2} \mathrm{O}_{2}: \mathrm{C} 72.72 \mathrm{H} 4.58 \mathrm{~N} \mathrm{10.60}$. Found: C 72.68 H 4.53 N 10.55 .

3-Hydroxy-3-(2-methyl-1H-indol-3-yl)indolin-2-one (3b). White solid; mp:176-178 ${ }^{\circ} \mathrm{C}$; $\mathrm{R}_{\mathrm{f}}$ 0.25 (50\% AcOEt/Petroleum ether); IR (neat): 3504, 3395, 3209, 1708, 1621, 1469, $755 \mathrm{~cm}^{-1} ;{ }^{1} \mathrm{H}$ nmr (500 MHz, DMSO-d 6$): \delta 2.36(3 \mathrm{H}, \mathrm{s}), 6.24$ (1 H, s, OH, $\mathrm{D}_{2} \mathrm{O}$ exchangeable), 6.69 (1 H, t, $J$ 
7.65), 6.85-6.90 (4 H, m), 7.13-7.18 (3 H, m), $10.31(1 \mathrm{H}, \mathrm{s}, \mathrm{NH}), 10.84(1 \mathrm{H}, \mathrm{s}, \mathrm{NH}) ;{ }^{13} \mathrm{C} \mathrm{nmr}$

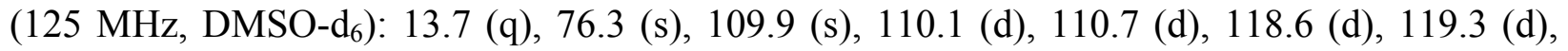
119.7 (s), 120.3 (d), 122.2 (d), 125.4 (d), 127.1 (s), 129.5 (d), 134.6 (s), 135.3 (s), 142.0 (s), 179.1 (s); MS (EI): $\mathrm{m} / \mathrm{z}=279.11\left[\mathrm{M}^{+}\right]$. Anal. Calcd for $\mathrm{C}_{17} \mathrm{H}_{14} \mathrm{~N}_{2} \mathrm{O}_{2}$ : C 73.37 H 5.07 N 10.07. Found: C $73.31 \mathrm{H} 5.02 \mathrm{~N} 10.01$.

3-Hydroxy-5-methoxy-3-(1H-indol-3-yl)indolin-2-one (3c). White solid; mp: 196-198 ${ }^{\circ} \mathrm{C}$; $\mathrm{R}_{\mathrm{f}}$ 0.25 (50\% AcOEt/Petroleum ether); IR (neat): 3326, 1721, 1619, 1472, 1177, $760 \mathrm{~cm}^{-1} ;{ }^{1} \mathrm{H} \mathrm{nmr}$ (500 MHz, DMSO-d 6 ): $\delta 3.58(3 \mathrm{H}, \mathrm{s}), 6.32\left(1 \mathrm{H}, \mathrm{s}, \mathrm{OH}, \mathrm{D}_{2} \mathrm{O}\right.$ exchangeable), 6.67 (1 H, d, J 8.4), 6.81 (1 H, s, ), 6.88 (1 H, d, J 8.4), 6.93-6.97 (2 H, m), 7.19-7.23 (3 H, m), 10.30 (1 H, s, $\mathrm{NH}), 10.81$ (1 H, s, NH); ${ }^{13} \mathrm{C} \mathrm{nmr}\left(125 \mathrm{MHz}, \mathrm{DMSO}_{\mathrm{d}}\right): 55.1$ (q), 74.9 (s), 102.7 (d), 109.5 (d), 110.8 (d), 112.0 (d), 115.0 (s), 121.7 (d), 124.2 (d), 124.8 (d), 125.3 (s), 129.0 (d), 132.0 (s), 133.3 (s), 141.7 (s), 152.7 (s), 178.4 (s); MS (EI): m/z = $295\left[\mathrm{M}^{+}\right]$; Anal. Calcd for $\mathrm{C}_{17} \mathrm{H}_{14} \mathrm{~N}_{2} \mathrm{O}_{3}$ : C 69.38 H 4.79 N 9.52. Found: C 69.16 H 4.75 N 9.47.

3-(5-Bromo-1H-indol-3-yl)-3-hydroxyindolin-2-one (3d). White solid; mp: 314-316 ${ }^{\circ} \mathrm{C}$, $\mathrm{R}_{\mathrm{f}}$ 0.25 (50\% AcOEt/Petroleum ether); IR (neat): $3411,3310,2924,1723 \mathrm{~cm}^{-1} ;{ }^{1} \mathrm{H} \mathrm{nmr}(500 \mathrm{MHz}$, DMSO-d $\left.{ }_{6}\right): \delta 6.25\left(1 \mathrm{H}, \mathrm{s}, \mathrm{OH}, \mathrm{D}_{2} \mathrm{O}\right.$ exchangeable), 7.02-7.07 (3 H, m), 7.36-7.4 (4 H, m), 7.83 (1 H, d, J 2.22), 10.16 (1 H, s, NH), $10.93(1 \mathrm{H}, \mathrm{s}) ;{ }^{13} \mathrm{C}$ nnmr (125 MHz, DMSO-d 6 ): 75.1 (s), 110.2 (d), 111.6 (s), 114.0 (d), 115.7 (s), 122.3 (d), 123.6 (d), 124.1 (d), 125.2 (d), 125.6 (d), 127.4 (s), 129.7 (d), 133.3 (s), 136.0 (s), 142.1 (s), 178.7 (s); MS (EI): m/z = 343 [M $\left.{ }^{+}\right], 345$ $\left[\mathrm{M}^{+2}\right]$. Anal. Calcd for $\mathrm{C}_{16} \mathrm{H}_{11} \mathrm{BrN}_{2} \mathrm{O}_{2}$ : C $56.00 \mathrm{H} 3.23 \mathrm{~N}$ 8.16. Found: C 56.27 H 3.34 N 8.08.

1-Allyl-3-hydroxy-3-(1H-indol-3-yl)indolin-2-one (3e). White solid; mp: $148-150^{\circ} \mathrm{C} ; \mathrm{R}_{\mathrm{f}} 0.25$ (50\% AcOEt/Petroleum ether); IR (neat): 3297, 1698, 1610, 1463, 1369, $742 \mathrm{~cm}^{-1} ;{ }^{1} \mathrm{H} \mathrm{nmr}(500$ MHz, DMSO-d $\left.{ }_{6}\right): \delta$ 4.23-4.35 (2 H, m), 5.13-5.2 (2 H, m) 5.8-5.86 (1 H, m) $6.49(1 \mathrm{H}, \mathrm{s}, \mathrm{OH}$, $\mathrm{D}_{2} \mathrm{O}$ exchangeable), $6.84(1 \mathrm{H}, \mathrm{t}, J$ 6.9), 6.98-7.03 $(4 \mathrm{H}, \mathrm{m}), 7.28-7.33(4 \mathrm{H}, \mathrm{m}), 11.00(1 \mathrm{H}, \mathrm{s}$, $\mathrm{NH}) ;{ }^{13} \mathrm{C} \mathrm{nmr}\left(125 \mathrm{MHz}, \mathrm{DMSO}-\mathrm{d}_{6}\right): 41.9$ (t), 75.1 (s), 109.6 (d), 112.0 (t), 115.6 (s), 117.5 (d), 119.0 (d), 120.9 (d), 121.6 (d), 122.8 (d), 124.1 (d), 125.0 (d), 125.4 (s), 127.3 (d), 129.5 (d), 132.4 (s), 133.2 (s), 142.7 (s), 176.9 (s); MS (EI): $\mathrm{m} / \mathrm{z}=305.02\left[\mathrm{M}^{+}\right]$; Anal. Calcd for $\mathrm{C}_{19} \mathrm{H}_{16} \mathrm{~N}_{2} \mathrm{O}_{2}$ : C $74.98 \mathrm{H} 5.30 \mathrm{~N}$ 9.20. Found: C 74.92 H 5.24 N 9.15.

1-Allyl-3-hydroxy-3-(2-methyl-1H-indol-3-yl)indolin-2-one (3f). White solid; mp: 164-166 ${ }^{\circ}$; $\mathrm{R}_{\mathrm{f}} 0.25$ (50\% AcOEt/Petroleum ether); IR (neat): 3433, 3320, 1705, 1617, 1371, 1176, $744 \mathrm{~cm}^{-1}$; ${ }^{1} \mathrm{H}$ nmr (500 MHz, DMSO-d 6 ): $\delta 3.03$ (3 H, s) 4.24-4.36 (2 H, m), 5.14-5.2 (2 H, m), 5.80-5.84 $(1 \mathrm{H}, \mathrm{m}), 6.40\left(1 \mathrm{H}, \mathrm{s}, \mathrm{OH}, \mathrm{D}_{2} \mathrm{O}\right.$ exchangeable), 6.7 (1 H, t, J 6.9), 6.83-6.9 $(2 \mathrm{H}, \mathrm{m}), 6.98(2 \mathrm{H}$, t, J 7.7), 7.17 (1 H, d, J 8.45), 7.24 (1 H, d, J 6.9), 7.27 (1 H, t, J 10.7), $10.89(1 \mathrm{H}, \mathrm{s}, \mathrm{NH}) ;{ }^{13} \mathrm{C}$ nmr (125 MHz, DMSO-d $)$ ): 13.4 (q), 41.9 (t), 76.0 (s), 109.6 (d), 110.4 (t), 115.4 (d), 116.4 (s), 118.2 (d), 120.3 (d), 122.9 (d), 125.2 (d), 127.0 (s), 129.5 (d), 130.4 (d), 133.9 (s), 134.2 (s), 135.3 (s), 142.7 (s), 177.1 (s); MS (EI): m/z = $319\left[\mathrm{M}^{+}\right]$. Anal. Calcd for $\mathrm{C}_{20} \mathrm{H}_{18} \mathrm{~N}_{2} \mathrm{O}_{2}: \mathrm{C} 75.45 \mathrm{H}$ 5.70 N 8.80. Found: C 75.40 H 5.63 N 8.83.

1-Allyl-3-hydroxy-3-(5-bromo-1H-indol-3-yl)indolin-2-one (3g). White solid; mp: 136-138 ${ }^{\circ}$; $\mathrm{R}_{\mathrm{f}} 0.25$ (50\% AcOEt/Petroleum ether); IR (neat): 3287, 1697, 1610, 1465, 1370, 1104, $754 \mathrm{~cm}^{-1}$; ${ }^{1} \mathrm{H}$ nmr (500 MHz, DMSO-d $\left.{ }_{6}\right): \delta$ 4.18-4.33 (2 H, m), 5.13 (2 H, t, J 10.7), 5.77-5.83 (1 H, m), 
6.54 (1 H, s, OH, $\mathrm{D}_{2} \mathrm{O}$ exchangeable), $6.93(1 \mathrm{H}, \mathrm{s}), 6.99(1 \mathrm{H}, \mathrm{d}, J$ 7.65), 7.05 (1 H, t, J 8.4), 7.14 (1 H, d, J 6.9), 7.28-7.32 (3 H, m), $7.70(1 \mathrm{H}, \mathrm{s}), 11.19(1 \mathrm{H}, \mathrm{s}, \mathrm{NH}) ;{ }^{13} \mathrm{C} \mathrm{nmr}(125 \mathrm{MHz}$, DMSO-d $)_{6}$ ) 41.9 (t), 74.9 (s), 109.7 (d), 110.9 (t), 114.5 (d), 115.5 (s), 117.5 (d), 122.9 (d), 123.7 (d), 124.2 (d), 125.0 (d), 125.6 (s), 127.4 (s), 129.8 (d), 132.3 (d), 132.6 (s), 136.0 (s), 142.6 (s), 176.6 (s); MS (EI): m/z = $383\left[\mathrm{M}^{+}\right], 385\left[\mathrm{M}^{+2}\right]$. Anal. Calcd for $\mathrm{C}_{19} \mathrm{H}_{15} \mathrm{BrN}_{2} \mathrm{O}_{2}$ : C $59.55 \mathrm{H} 3.95$ N 7.31. Found: C 59.50 H 3.91 N 7.27.

1-Benzyl-3-hydroxy-3-(1H-indol-3-yl)indolin-2-one (3h). White solid; mp: $120-124^{\circ} \mathrm{C} ; \mathrm{R}_{\mathrm{f}} 0.25$ (50\% AcOEt/Petroleum ether); IR (neat): 3401, 1706, 1611, 1488, 1462, 1351, 1173, $746 \mathrm{~cm}^{-1}$; ${ }^{1} \mathrm{H}$ nmr (500 MHz, DMSO-d 6 ): $\delta 4.89$ (2 H, ABq, J 16.1), 6.57 (1 H, s, OH, D $\mathrm{O}_{2}$ exchangeable), 6.79 (1 H, t, J 7.65), 6.94-7.02 (3 H, m), 7.08 (1 H, s), 7.23-7.33 (9 H, m), $11.02(1 \mathrm{H}, \mathrm{s}, \mathrm{NH})$; ${ }^{13} \mathrm{C}$ nmr (125 MHz, DMSO-d $\left._{6}\right): 43.3$ (t), 75.2 (s), 109.2 (d), 112.0 (d), 115.7 (s), 118.4 (d), 120.3 (d), 121.1 (d), 121.5 (d), 123.7 (d), 124.1 (d), 125.0 (d), 127.3 (s), 128.1 (d), 129.0 (d), 130.3 (s), 133.2 (d), 136.8 (s), 137.3 (s), 142.6 (s), 177.3 (s); MS (EI): m/z = $355\left[\mathrm{M}^{+}\right]$.Anal. Calcd for $\mathrm{C}_{23} \mathrm{H}_{18} \mathrm{~N}_{2} \mathrm{O}_{2}$ : C 77.95 H 5.12 N 7.90. Found: C 77.89 H 5.07 N 7.85.

1-Benzyl-3-hydroxy-3-(2-methyl-1H-indol-3-yl)indolin-2-one (3i). White solid; mp: 96-98 ${ }^{\circ}$; $\mathrm{R}_{\mathrm{f}} 0.25$ (50\% AcOEt/Petroleum ether); IR (neat): 3397, 2363, 1707, 1612, 1461, 1366, 1173, 747 $\mathrm{cm}^{-1}$; ${ }^{1} \mathrm{H}$ nmr (500 MHz, DMSO-d 6 ): $\delta 2.35$ (3 H, s), $4.89(2 \mathrm{H}, \mathrm{ABq}, J 16.1) 6.47(1 \mathrm{H}, \mathrm{s}, \mathrm{OH}$, $\mathrm{D}_{2} \mathrm{O}$ exchangeable), $6.64(1 \mathrm{H}, \mathrm{t}, J$ 7.65), $6.76(1 \mathrm{H}, \mathrm{d}, J$ 8.4) 6.88 (1 H, t, J 7.6 ), 6.96 (2 H, t, J 8.4 ), 7.16-7.35 (8 H, m) 10.9 (1 H, s, NH); ${ }^{13} \mathrm{C} \mathrm{nmr}\left(125 \mathrm{MHz}, \mathrm{DMSO}-\mathrm{d}_{6}\right): 13.8$ (q), 43.2 (t), 76.1 (s), 109.8 (d), 109.7 (d), 110.8 (s), 118.6 (d), 119.7 (d), 120.3 (d), 123.0 (s), 125.3 (d), 127.0 (d), 127.7 (d), 128.0 (d), 128.9 (d), 129.1 (d), 129.5 (d), 133.9 (s), 134.2 (s), 135.3 (s), 136.8 (s), 142.6 (s), 177.5 (s); MS (EI): m/z = $369\left[\mathrm{M}^{+}\right]$. Anal. Calcd for $\mathrm{C}_{24} \mathrm{H}_{20} \mathrm{~N}_{2} \mathrm{O}_{2}:$ C $78.24 \mathrm{H} 5.47 \mathrm{~N}$ 7.60. Found: C 78.20 H 5.42 N 7.53.

1-Benzyl-3-hydroxy-3-(5-methoxy-1H-indol-3-yl)indolin-2-one (3j). White solid; mp: 204$206^{\circ} \mathrm{C} ; \mathrm{R}_{\mathrm{f}} 0.25$ (50\% AcOEt/Petroleum ether); IR (neat): 3424, 1703, 1610, 1462, 1367, 1212, 1172, 1073, 802, $751 \mathrm{~cm}^{-1}$; ${ }^{1} \mathrm{H}$ nmr (500 MHz, DMSO-d 6 ): $\delta 3.47(3 \mathrm{H}, \mathrm{s}), 4.88(2 \mathrm{H}, \mathrm{ABq}, J$ 16.0) 6.54 (1 H, s, OH, $\mathrm{D}_{2} \mathrm{O}$ exchangeable), 6.67 (2 H, d, J 12.25) 6.94 (1 H, d, J 7.65), 7.01 (2 $\mathrm{H}, \mathrm{t}, J$ 6.9), 7.2-7.3 (8 H, m), $10.86(1 \mathrm{H}, \mathrm{s}, \mathrm{NH}) ;{ }^{13} \mathrm{C} \mathrm{nmr}\left(125 \mathrm{MHz}, \mathrm{DMSO}-\mathrm{d}_{6}\right): 43.1$ (t), 55.4 (q), 75.2 (s), 102.6 (d), 109.6 (d), 111.7 (d), 112.6 (d), 115.1 (s), 123.1 (s), 124.8 (d), 125.1 (s), 125.7 (s), 127.8 (d), 127.9 (d), 129.0 (d), 129.6 (d), 132.4 (d), 133.1 (s), 136.8 (d), 142.6 (s), 153.2 (s), 177.3 (s); MS (EI): m/z $=385\left[\mathrm{M}^{+}\right]$. Anal. Calcd for $\mathrm{C}_{24} \mathrm{H}_{20} \mathrm{~N}_{2} \mathrm{O}_{3}: \mathrm{C} 74.98 \mathrm{H} 5.24 ; \mathrm{N}$ 7.29. Found: C 74.92 H 5.19 N 7.22.

1-Benzyl-3-hydroxy-3-(5-bromo-1H-indol-3-yl)indolin-2-one (3k). White solid; mp: 150$152^{\circ} \mathrm{C} ; \mathrm{R}_{\mathrm{f}} 0.25$ (50\% AcOEt/Petroleum ether); IR (neat): 3265, 1707, 1616, 1463, 1377, $744 \mathrm{~cm}^{-}$ ${ }^{1},{ }^{1} \mathrm{H} \mathrm{nmr}\left(500 \mathrm{MHz}, \mathrm{DMSO}_{6}\right): \delta 4.84(2 \mathrm{H}, \mathrm{ABq}, J 16.1), 6.63\left(1 \mathrm{H}, \mathrm{s}, \mathrm{OH}, \mathrm{D}_{2} \mathrm{O}\right.$ exchangeable), $6.93(2 \mathrm{H}, \mathrm{d}, J$ 8.4), 6.97 (1 H, d, J 2.25), 7.04 (1 H, t, J 7.65), 7.15 (1 H, d, J 6.9), 7.25-7.33 (7 H, m), 7.67 (1 H, d, J 2.3), 11.23 (1 H, s, NH); ${ }^{13} \mathrm{C} \mathrm{nmr}\left(125 \mathrm{MHz}, \mathrm{DMSO}-\mathrm{d}_{6}\right)$ : $43.1(\mathrm{t}), 74.9$ (s), 109.8 (d), $111.2(\mathrm{~s}), 114.6$ (d), 115.4 (s), 123.1 (s), 123.7 (d), 124.2 (d), 125.1 (d), 125.8 (d), 127.4 (s), 127.7 (d), 127.9 (d), 129.1 (d), 129.8 (d), 132.6 (s), 136.1 (s), 136.8 (d), 
142.6 (s), 177.0 (s); MS (EI): m/z = $433\left[\mathrm{M}^{+}\right], 435\left[\mathrm{M}^{+2}\right]$. Anal. Calcd for $\mathrm{C}_{23} \mathrm{H}_{17} \mathrm{BrN}_{2} \mathrm{O}_{2}: \mathrm{C}$ 63.75 H 3.95 N 6.47. Found: C 63.70; H 3.89 N 6.40.

5-Chloro-3-hydroxy-3-(1H-indol-3-yl)indolin-2-one (3l). White solid; mp: 206-208 ${ }^{\circ}$; $\mathrm{R}_{\mathrm{f}} 0.25$ (50\% AcOEt/Petroleum ether); IR (neat): 3422, 3357, 1731, 1620, 1476, 1178, $749 \mathrm{~cm}^{-1} ;{ }^{1} \mathrm{H} \mathrm{nmr}$ (500 MHz, DMSO-d 6 ): $\delta 6.51\left(1 \mathrm{H}, \mathrm{s}, \mathrm{OH}, \mathrm{D}_{2} \mathrm{O}\right.$ exchangeable), 6.9-6.91 (2 H, m), 7.01 (1 H, t, $J$ 8.4), 7.08 (1 H, d, J 3.1), 7.18 (1 H, d, J 3.1), 7.28 (1 H, d, J 8.4), 7.33 (2 H, t, J 6.85), 10.47 (1 $\mathrm{H}, \mathrm{s}, \mathrm{NH},), 11.01$ (1 H, s, NH); ${ }^{13} \mathrm{C}$ nmr (125 MHz, DMSO-d 6 ): 75.5 (s), 111.7 (d), 112.1 (d), $115.1(\mathrm{~s}), 119.1$ (d), 120.5 (d), 121.7 (d), 124.1 (d), 125.08 (d) 125.2 (s), 126.2 (s), 129.4 (d),

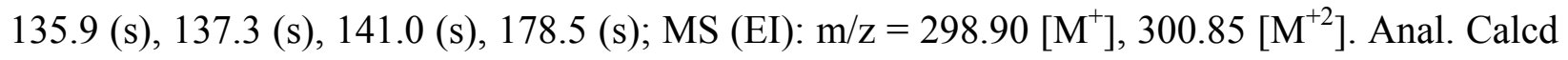
for $\mathrm{C}_{16} \mathrm{H}_{11} \mathrm{ClN}_{2} \mathrm{O}_{2}$ : C 64.33 H 3.71 N 9.38. Found: C 64.26 H 3.65 N 9.32.

5-Chloro-3-hydroxy-3-(2-methyl-1H-indol-3-yl)indolin-2-one (3m). White solid; mp: 174$176^{\circ} \mathrm{C} ; \mathrm{R}_{\mathrm{f}} 0.25$ (50\% AcOEt/Petroleum ether); IR (neat): 3341, 1711, 1617, 1465, 1164, $749 \mathrm{~cm}^{-}$ ${ }^{1}$; ${ }^{1} \mathrm{H} \mathrm{nmr}\left(500 \mathrm{MHz}, \mathrm{DMSO}-\mathrm{d}_{6}\right): \delta 2.39(3 \mathrm{H}, \mathrm{s}), 6.44\left(1 \mathrm{H}, \mathrm{s}, \mathrm{OH}, \mathrm{D}_{2} \mathrm{O}\right.$ exchangeable), 6.74 (1 $\mathrm{H}, \mathrm{t}, J$ 7), 6.88-6.93 (3 H, m), 7.11 (1 H, d, J 1.5), 7.18 (1 H, d, J 8.4), 7.26 (1 H, d, J 6.1), 10.5 (1 H, s, NH), 10.9 (1 H, s, NH). ${ }^{13} \mathrm{C} \mathrm{nmr}\left(125 \mathrm{MHz}, \mathrm{DMSO}-\mathrm{d}_{6}\right): 13.7$ (q), 76.4 (s), 109.1 (s), 110.9 (d), 111.7 (d), 118.9 (d), 119.3 (s), 120.4 (d), 125.2 (d), 126.2 (s), 126.9 (s), 129.3 (d), 134.1 (s), 135.3 (s), 136.6 (d), 140.9 (s), 178.7 (s); MS (EI): m/z = 312.82 [M $\left.{ }^{+}\right], 314$ [M $\left.{ }^{+2}\right]$. Anal. Calcd for $\mathrm{C}_{17} \mathrm{H}_{13} \mathrm{ClN}_{2} \mathrm{O}_{2}$ : C $65.29 \mathrm{H} 4.19 \mathrm{~N}$ 8.96. Found: $\mathrm{C} 65.23 \mathrm{H} 4.12 \mathrm{~N} 8.91$.

5-Chloro-3-hydroxy-3-(5-methoxy-1H-indol-3-yl)indolin-2-one (3n). White solid; mp: 208$210^{\circ} \mathrm{C} ; \mathrm{R}_{\mathrm{f}} 0.25$ (50\% AcOEt/Petroleum ether); IR (neat): 3301, 1712, 1622, 1476, 1216, 1170 $\mathrm{cm}^{-1}$; ${ }^{1} \mathrm{H} \mathrm{nmr}\left(500 \mathrm{MHz}, \mathrm{DMSO}-\mathrm{d}_{6}\right): \delta 3.61(3 \mathrm{H}, \mathrm{s}), 6.5$ (1 H, s, OH, $\mathrm{D}_{2} \mathrm{O}$ exchangeable), 6.7 (1 $\mathrm{H}, \mathrm{d}, J$ 8.4), 6.84 (1 H, d, J 2.3), 6.9 (1 H, d, J 8.4), 7.01 (1 H, d, J 2.3), 7.21 (2 H, t, J 8.4), 7.29 (1 H, d, J 8.4), 10.47 (1 H, s, NH), 10.87 (1 H, s, NH); ${ }^{13} \mathrm{C} \mathrm{nmr}\left(125 \mathrm{MHz}, \mathrm{DMSO}_{6} \mathrm{~d}_{6}\right): 55.7$ (t), $75.5(\mathrm{~s}), 102.9$ (s), 111.4 (d), 111.6 (d), 112.7 (d), 114.7 (d), 124.8 (d), 125.2 (d), 125.6 (s), 126.2 (d), 129.3 (d), 132.5 (d), 135.8 (s), 141.0 (s), 153.3 (s), 178.5 (s); MS (EI): m/z = 328.93 [M ], $330.82\left[\mathrm{M}^{+2}\right]$. Anal. Calcd for $\mathrm{C}_{17} \mathrm{H}_{13} \mathrm{ClN}_{2} \mathrm{O}_{3}$ : C 62.11 H 3.99 N 8.52. Found: C 62.07 H $3.92 \mathrm{~N}$ 8.46 .

11-(1H-Indol-3-yl)-11H-indeno[1,2-b]quinoxalin-11-ol (5a). Yellow solid; mp: $250-252^{\circ} \mathrm{C} ; \mathrm{R}_{\mathrm{f}}$ 0.25 (50\% AcOEt/Petroleum ether); IR (neat): 3246, 1335, 1097, $759 \mathrm{~cm}^{-1} ;{ }^{1} \mathrm{H} \mathrm{nmr}\left(\mathrm{DMSO}-\mathrm{d}_{6}\right.$, $500 \mathrm{MHz}): \delta 6.61\left(1 \mathrm{H}, \mathrm{s}, \mathrm{OH}, \mathrm{D}_{2} \mathrm{O}\right.$ exchangeable), $6.64(1 \mathrm{H}, \mathrm{t}, J$ 7.65), $6.77(1 \mathrm{H}, \mathrm{d}, J$ 7.65), 6.90 (1 H, t, J 7.65), 7.27 (1 H, d, J 8.45), 7.34 (1 H, d, J 2.3), 7.51-7.55 (3 H, m), 7.68 (1 H, t, $J$ 8.4), 7.75 (1 H, t, J 8.4), 7.93 (1 H, d, J 8.4), 8.10 (2 H, t, J 8.4), $11.03\left(1 \mathrm{H}, \mathrm{s}, \mathrm{NH}\right.$,); ${ }^{13} \mathrm{C} \mathrm{nmr}$ (DMSO-d 6,75 MHz): 77.1 (s), 111.5 (s), 116.4 (s), 118.4 (d), 119.2 (d), 120.8 (d), 121.6 (s), 123.6 (d), 124.6 (s), 125.6 (s), 128.7 (d), 129.1 (s), 129.7 (d), 129.8 (d), 132.3 (d), 135.3 (d), 136.7 (s), 141.1 (d), 141.9 (d), 152.1 (d), 153.2 (s), 164.5 (s); MS (EI): m/z = 350 [M ]; Anal. Calcd for $\mathrm{C}_{23} \mathrm{H}_{15} \mathrm{~N}_{3} \mathrm{O}$ : C 79.07 H 4.33 N 12.03. Found: C 79.25 H 4.29 N 11.97.

11-(5-Methoxy-1H-indol-3-yl)-11H-indeno[1,2-b]quinoxalin-11-ol (5b). Yellow solid; mp: 214-216 ${ }^{\circ} \mathrm{C}$; $\mathrm{R}_{\mathrm{f}} 0.25$ (50\% AcOEt/Petroleum ether); IR (neat): 3265, 1356, $1123,819 \mathrm{~cm}^{-1} ;{ }^{1} \mathrm{H}$ nmr (DMSO-d $6,500 \mathrm{MHz}): \delta 3.48(3 \mathrm{H}, \mathrm{s}), 6.48$ (1 H, s, OH, $\mathrm{D}_{2} \mathrm{O}$ exchangeable), 6.59-6.61 (2 $\mathrm{H}, \mathrm{m}), 7.16-7.19$ (2 H, m), 7.57-7.61 (2 H, m), 7.62-7.65 (1 H, m), 7.71 (1 H, t, J 7.45), 7.76 (1 
H, t, J 7.45), 7.96 (1 H, d, J 7.45), 8.1-8.14 (2 H, m), $10.85(1 \mathrm{H}, \mathrm{s}) ;{ }^{13} \mathrm{C}$ nmr (125 MHz, DMSO$\left.\mathrm{d}_{6}\right): 55.5(\mathrm{q}), 77.8$ (s), 102.5 (d), 111.3 (d), 112.1 (s), 113.1 (s), 116.6 (s), 122.1 (s), 124.52 (s), 125.7 (d), 126.2 (s), 129.2 (s), 129.7 (d), 129.9 (d), 130.3 (d), 132.4 (d), 133.1 (d), 136.1 (s), 141.5 (d), 142.6 (d), 152.5 (d), 153.1 (d), 153.7 (s), 165.10 (s); MS (EI): m/z = 380 [M ]; Anal. Calcd for $\mathrm{C}_{24} \mathrm{H}_{17} \mathrm{~N}_{3} \mathrm{O}_{2}$ : C 75.97 H 4.52 N 11.08. Found: C 76.15 H 4.39 N 10.97.

11-(5-Bromo-1H-indol-3-yl)-11H-indeno[1,2-b]quinoxalin-11-ol (5c). Yellow solid; mp: 264$266^{\circ} \mathrm{C} ; \mathrm{R}_{\mathrm{f}} 0.25$ (50\% AcOEt/Petroleum ether); IR (neat): 3289, 1334, 1077, $754 \mathrm{~cm}^{-1} ;{ }^{1} \mathrm{H} \mathrm{nmr}$ (DMSO-d $6,500 \mathrm{MHz}): \delta 6.65\left(1 \mathrm{H}, \mathrm{s}, \mathrm{OH}, \mathrm{D}_{2} \mathrm{O}\right.$ exchangeable), $6.70(1 \mathrm{H}, \mathrm{t}, J$ 7.65), $6.8(1 \mathrm{H}, \mathrm{d}$, $J$ 7.65), 7.32 (1 H, d, J 8.45), 7.38 (1 H, d, J 2.3), 7.58-7.62 (3 H, m), 7.72 (1 H, t, J 8.4), 7.82 (1 $\mathrm{H}, \mathrm{t}, J$ 8.4), 8.1 (1 H, d, J 8.4), 8.18 (2 H, t, J 8.4), 11.06 (1 H, s, NH,); ${ }^{13} \mathrm{C} \mathrm{nmr}\left(\right.$ DMSO-d $_{6}, 75$ MHz): 77.7 (s), 111.8 (s), 115.8 (s), 118.1 (d), 119.8 (d), 121.5 (d), 121.6 (s), 124.2 (s), 125.3 (s), 125.6 (d), 127.6 (d), 128.7 (d), 129.4 (s), 129.7 (s), 131.3 (d), 134.7 (d), 135.8 (s), 141.6 (d), 142.2 (d), 152.1 (d), 153.8 (s), 165.5 (s); MS (EI): m/z = $429\left[\mathrm{M}^{+}\right], 431\left[\mathrm{M}^{+2}\right]$; Anal. Calcd for $\mathrm{C}_{23} \mathrm{H}_{14} \mathrm{~N}_{3} \mathrm{O}$ : C $64.50 \mathrm{H} 3.29 \mathrm{~N}$ 9.81. Found: C 64.61 H 3.23 N 9.76.

\section{Acknowledgements}

One of the authors NV thanks UGC and CSIR (New Delhi) for the award of Junior Research Fellowship.

\section{References}

1. Kamano, Y.; Zhang, H. P.; Ichihara Y.; Kizu, H.; Komiyama, K.; Pettit,G. R. Tetrahedron Lett. 1995, 36, 2783 .

2. Rasmussen, H. B.; MacLeod, J. K. J. Nat. Prod. 1997, 60, 1152.

3. Balk-Bindseil, W.; Helmke, E.; Weyland, H.; Laatsch, H.; Liebigs Ann. 1995, 1291.

4. Monde, K.; Sasaki, K.; Shirata, A.; Tagusuki, M. Phytochemistry 1991, 30, 2915

5. Suzuki, H,; Morita, H.; Shiro, M.; Kobayashi, M. Tetrahedron 2004, 60, 2489

6. Frechard, A.; Fabre, N.; Pean, C.; S. Montaut, S.; Fauvel, M. T.; Rollin, P.; Fouraste, I. Tetrahedron Lett. 2001, 42, 9015.

7. Kohno, J.; Koguchi, Y.; Nishio, M.; Nakao, K.; Kuroda, M.; Shimizu, R.; Ohnuki, T.; Komatsubara, S. J. Org. Chem. 2000, 65, 990.

8. (a) Hewawasam, P.; Meanwell, N. A.; Gribkoff, V. K.; Dworetzky, S. I.; Biossard, C. G. Bioorg. Med. Chem. Lett. 1997, 7, 1255. (b) Hewawasam, P.; Erway, M.; Moon, S. L.; Knippe, J.; Weiner, H.; Biossard, C. G.; Post-Munson, D. J.; Gao, Q.; Huang, S.; Gribkoff, V. K.; Meanwell, N. A. J. Med. Chem. 2002, 45, 1487.

9. (a) Natarajan, A.; Fam, Y. H.; Chen, H.; Guo, Y.; Iyasere, J.; Harbinski, F.; Christ, W. J.; Aktas, H.; Halperin, J. A. J. Med. Chem. 2004, 47, 1882. (b) Cliffe, I. A.; Lien, E. L.; 
Mansell, H. L.; Steiner, K. E.; Todd, R. S.; White, A. C.; Black, R. M. J. Med. Chem. 1992, 35, 1169.

10. (a) Narayan, S.; Muldoon, J.; Finn, M. G.; Fokin, V. V.; Kolb, H. C.; Sharpless, K. B. Angew. Chem., Int. Ed. 2005, 44, 3275. (b) Li, C -J. Chem. Rev. 2005, 105, 3095. (c) Kavala, V.; Samal. A. K.; Patel, B. K. ARKIVOC 2005, (i), 20. (d) Hui, A.; Xu, X.; Zha, Z.; Zhou, C.; Wang, Z. ARKIVOC 2004, (ix), 52. (e) Gupta, M.; Wakhloo, B. P. ARKIVOC 2007, (i), 94. (f) Tohma, H.; Maegawa, T.; Kita, Y. ARKIVOC 2003, (vi) 62. (g) Wang, G. W.; Cheng, B. ARKIVOC 2004, (ix), 4. (h) Jin, T. S.; Zhao. R. Q.; Li, T. S.; ARKIVOC 2006, (xi), 176. (i) Amantini, D.; Fringuelli, F.; Piermatti, O.; Tortoioli, S.; Vaccaro, L.; ARKIVOC 2002, (xi), 293. (j) Naik, S.; Bhattacharjya, G.; Kavala, V. R.; Patel, B. K. ARKIVOC 2004, (i), 55. 11. Wang, S. Y.; Ji, S. J. Tetrahedron 2006, 62, 1527.

12. (a) Musabekova, Z. R.; Gurevich, P. A.; Zykova, T.V.: Zhurnal Obshchei Khimii 1994, 64, 254. (b) Kumar, V. P.; Reddy, V.P.; Sridhar, R.; Srinivas, B.; Narender, M.; Rama Rao, K. J. Org. Chem., 2008, 73, 1646.

13. (a) Shanthi, G.; Subbulakshmi, G.; Perumal, P. T. Tetrahedron 2007, 63, 2057. (b) Shanthi, G.; Perumal, P. T. Tetrahedron Lett. 2007, 48, 6785. (c) Savitha, G.; Niveditha, S. K.; Muralidharan, D.; Perumal, P. T. Tetrahedron Lett. 2007, 48, 2943. (d) Selvam, N. P.; Shanthi, G.; Perumal, P. T. Can. J. Chem. 2007, 85, 989.

14. (a) Azizian, J.; Mohammadizadeh, M. R.; Zomorodbakhsh, S.; Mohammadi, A. A.; Karimi, A. R. ARKIVOC 2007, (xv), 24. (b) Islami, M. R.; Hassani, Z. ARKIVOC 2008, (xv), 280. (c) Hasaninejad, A.; Zare, A.; Mohammadizadeh, M. R.; Shekouhya, M. ARKIVOC 2008, (xiii), 28. (d) Heravi, M. M.; Bakhtiari, K.; Tehrani, M. H.; Javadi, N. M.; Oskooie, H. A. ARKIVOC 2006, (xvi), 16.

15. Azizian, J.; Ali, R. K.; Zahra, K.; Ali, A. M.; Mohammad, R. M. Tetrahedron Lett. 2005, 46, 6155.

16. Azizian, J.; Shaabanzadeh, M.; Hatamjafari, F.; Mohammadizadeh, M. R. ARKIVOC 2006, (xi), 47. 